\title{
Prominent Accessory Hemiazygos Vein representing Persistence of Cranial Portion of Posterior Cardinal Vein: Embryological Variant
}

\author{
Seema Sehmi
}

\begin{abstract}
Introduction: The azygos venous system has developed from the postcardinal veins of the embryo, and small parts persist only at the commencement of the azygos vein. Complex embryological development of these veins is a major cause for many possible variations of the azygos system.
\end{abstract}

Materials and methods: The present study was done in the Department of Anatomy, Government Medical College, Amritsar, on a male cadaver aged about 50 years. The thoracic cavity was opened to dissect the azygos system of veins.

Results: The system showed the persistent cranial part of the posterior cardinal vein. This led to the formation of a welldeveloped venous channel on the left side of the aortic arch and the thoracic aorta. This channel drained the 2nd to 10th left posterior intercostal veins and drained itself into the left brachiocephalic vein (LBV) after crossing the arch of aorta vertically on its right. There was no transvertebral communication between this channel and azygos vein on the right side. The hemiazygos vein (HAV) on the left side was normally formed and immediately drained into the azygos vein by turning to the left. The azygos vein on the right side was also normal.

Conclusion: The presence of such persistent embryological remnant, its position, and approximation to the structures near the arch of aorta should be taken into account to avoid radiological misdiagnosis and surgical complications.

Keywords: Azygos, Cardinal, Embryological, Hemiazygos.

How to cite this article: Sehmi S. Prominent Accessory Hemiazygos Vein representing Persistence of Cranial Portion of Posterior Cardinal Vein: Embryological Variant. Curr Trends Diagn Treat 2017;1(1):40-41.

Source of support: Nil

Conflict of interest: None

\section{INTRODUCTION}

Variations of the venous system enable clinicians to distinguish features that merit further investigations or

\section{Professor}

Department of Anatomy, Sri Guru Ram Das Institute of Medical Sciences and Research, Amritsar, Punjab, India

Corresponding Author: Seema Sehmi, Professor, Department of Anatomy, Sri Guru Ram Das Institute of Medical Sciences and Research, Amritsar, Punjab, India, Phone: +919914754354 e-mail: drseema16@gmail.com treatment from those that do not. ${ }^{1}$ So, each encounter of a practitioner with the anatomical variations should contribute to broaden his or her experiences that assist in the recognition of these boundaries. ${ }^{2}$ The variations of the azygos venous system are common. The hemiazygos is more variable than the azygos vein, but the accessory hemiazygos vein $(\mathrm{AHV})$ most variably drains in the brachiocephalic, azygos, or HAV. ${ }^{3}$ These deviations in the patterns are due to complex embryological development. ${ }^{4}$ The azygos system of veins arises from a pair of longitudinal venous lines called right and left azygos venous lines on each side of the aorta. On each side of the azygos venous lines lies two parallel primitive venous lines called the right and left subcardinal and posterior cardinal veins. The right azygos venous line develops into the vertical part of the azygos vein. The arch of azygos is contributed partially by persistent cephalic part of right posterior cardinal vein. caudal part of the left azygos line. Due to the development of an oblique communication between the right and the left anterior cardinal veins, the distal part of the left anterior cardinal vein, the cranial part of the left anterior cardinal vein, and the left common cardinal vein regress greatly. ${ }^{5}$ The left azygos line gives rise to AHV above and drains the 4th to 6th left posterior intercostal veins. The lower part gives rise to HAV draining 7th to 11th left posterior intercostal veins. ${ }^{6}$ The coexistence of the anomalies of the heart, spleen, and superior vena cava is reported to be associated with the variations of the azygos venous system, especially if associated with the azygos vein continuous with the inferior vena cava. ${ }^{7,8}$

\section{CASE REPORT}

During exposure of the posterior mediastinum a prominent AHZ vein was seen lying on the left side parallel to aorta. On further dissection, this channel was about $17 \mathrm{~cm}$ in length. It drained the 1st to 10th left posterior intercostal veins and drained itself vertically into the left brachiocephalic vein (LBV) (Fig. 1). The azygos vein, superior vena cava, and inferior vena cava were normal. There was no communication between this channel and azygos vein. The HAV was less developed and drained immediately into the azygos vein after its formation. The 


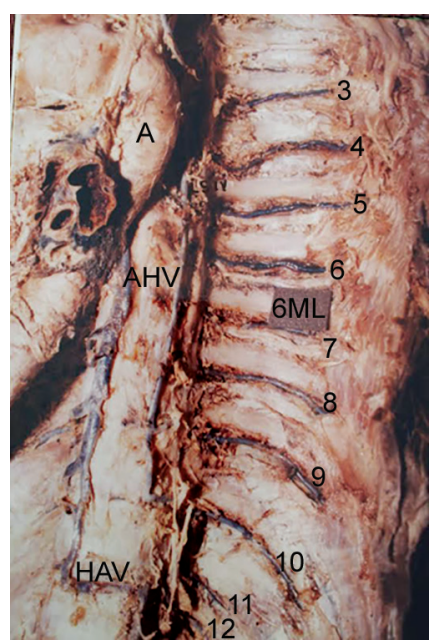

Fig. 1: Accessory hemiazygos vein draining upper 10 left posterior intercostal veins and opening into LBV. Hemiazygos vein draining into azygos vein. $A=$ Arch of aorta

cardiovascular system and the respiratory system showed no gross anomaly.

\section{DISCUSSION}

The complex embryological development causes many possible variations in the form of agenesis of one part and development of the other part, leading to redistribution of area of drainage of azygos, hemiazygos, and AHV. ${ }^{9}$ The present variation was also recorded by $\mathrm{Muhm}$ et $\mathrm{al}^{10}$ and Mahato, ${ }^{11}$ where the AHV instead of draining into the azygos vein drained into the LBV after joining the left superior intercostal vein. A complete reverse case was noted by Ozbek et al. ${ }^{5}$ The present variation can be explained on the basis of variation in the development of left azygos vein on the left side. The left superior intercostal vein ends in this long channel, and this channel drained into LBV. Embryologically, this could be explained by the persistence of the cranial part of the azygos line on the left and the oblique communication represented by the LBV. The present long channel represented the AHV with an added cranial extension into the remaining and persistent cephalic part of the left posterior cardinal vein. ${ }^{9}$ In the absence of the $\mathrm{HAV}$, the left superior intercostal vein formed the terminal conduit for the entire upper left posterior thorax for its venous drainage. ${ }^{12}$ It is important to interpret these variations while making diagnosis and to avoid complications during mediastinal procedures. ${ }^{13}$ Also, the confusion can be avoided in diagnosing these structures after detection in magnetic resonance imaging scans. ${ }^{14}$

\section{CONCLUSION}

The present variation is clinically important because of the position of long channel close to the left of the aortic arch. It can present radiologically as a small mass or nipple adjacent to the lateral or superior aspect of the arch seen best on supine anteroposterior mediastinal tomograms. This physiologically normal venous shadow should not be confused with lymphadenopathy or small tumor.

\section{REFERENCES}

1. Willan PLT, Humpherson JR. Concepts of variations and normality in morphology: important issues at risk of neglect in modern undergraduate medical courses. Clin Anat 1999;12(3):186-190.

2. Scherpbier AJJA, Faber V, Yspeerr-Getards J. The preclinical eye: do senior medical students know what is normal? Med Teach 1997;19(1):29-31.

3. Celix HH, Sargon MF, Aldur MM, Cumhur M. An anomalous course of the interazygos vein. Surg Radiol Anat 1996;18(1): 61-62.

4. Mezzogiorno A, Passiate C. An atypic pattern of the azygos venous system in man. Anat Anz 1998;165(5):277-281.

5. Ozbek A, Daleik C, Colak T. Multiple variations of the azygos venous system. Surg Radiol Anat 1999;21(1):83-85.

6. Williams, P.L.; Warwick, R.; Dyson, M.; Bannister, L.H. Heart and mediastinum. In: Gray's anatomy. 40th ed. London: Churchill Livingstone; 2008. p. 939-940.

7. Minniti S, Visentini S, Procacci C. Congenital anomalies of the venae cavae: embryological origin, imaging features and report of three new variants. Euro Radiol 2002 Aug;12(1): 2040-2055.

8. Vijayvergiya R, Bhat MN, Kumar RM, Vivekanand SG, Grover A. Azygos continuation of interrupted inferior vena cava in association with sick sinus syndrome. Heart 2005 Apr;91(4):26.

9. Seib GA. The azygos system of veins in American Whites and American Negroes. Am J Physiol Anthropol 1934 Apr;19(1);39-163.

10. Muhm M, Plassmann GS, Druml W. Malposition of the dialysis catheter in the accessory hemiazygos vein. Anesth Analg 1996 Oct;83(4):883-885.

11. Mahato NK. Anomalous accessory hemi-azygos system with persistent cranial segment of posterior cardinal vein - a case report. Braz J Morphol Sci 2009 Jan;26(3-4):177-179.

12. Ozdemir B, Aldur MM, Celix HH. Multiple variations of the azygos venous systems: a preaortic interazygos vein and absence of the hemiazygos vein. Surg Radiol Anat 2002 Feb;24(1):68-70.

13. Brenner BJ, Darling RC, Frederick PL, Linton RR. Major venous anomalies complicating abdominal aortic surgery. Arch Surg 1974 Feb;108(2):159-165.

14. Smather RL, Lee JKT, Heiken JP. Anomalous preaortic interazygos vein. J Comp Assist Tomogr 1983;7:732-733. 